\title{
Monitoring and testing corrosion and responsive behaviour in galvanic systems.
}

\author{
Christian Stone ${ }^{1}$, Gareth Glass ${ }^{1}$, Adrian Roberts ${ }^{1}$, and Nigel Davison ${ }^{1}$ \\ ${ }^{I}$ Concrete Preservation Technologies, Long Eaton, Nottinghamshire, UK
}

\begin{abstract}
Testing methods for corrosion prevention in structures have, historically, been based on the steel polarising from the protective current. Modern galvanic systems aim to protect the steel by restoring the protective alkaline environment, rather than through polarisation. Since 2012 standard guidelines allow for alternative testing to be used [1]. Scientific testing of galvanic systems will be discussed looking at the nature of constant potential galvanic systems and comparing them to the traditional constant current impressed systems, looking in particular at unique anode behaviours such as low median current during times of low corrosion risk and responsive behaviour during corrosion hazards. This work lays out a protocol for the evaluation and testing of existing galvanic corrosion protection $(\mathrm{CP})$ systems in reinforced concrete through the lens of various real-world studies. Data taken from over a decade of monitored systems are used to support the protocol. Modern testing strategies such as 2-dimensional relative potential mapping and corrosion rate monitoring will measure the steel's corrosion. Galvanic currents rise and fall with corrosion risk providing protection while ensuring a long life to the design.
\end{abstract}

\section{Introduction:}

Cathodic protection $(\mathrm{CP})$ in reinforced concrete structures has been used to control corrosion on bridge structures since the 1950s [2] [3]. The question of how to test and monitor these structures has been an area of concern from early on in part because many systems have no inbuilt monitoring devices. Existing galvanic systems are reaching or surpassing their estimated design life and knowledge of the ongoing corrosion is essential to the health of the protected structures. New systems also must be actively monitored to assess the effectiveness of the corrosion management.

Unlike typical impressed current systems which run at a constant current and aim to polarise the steel in order to halt corrosion [4], galvanic systems are electrochemical and hold a constant voltage. They supply current relative to changes in moisture, ion concentration and heat [5], with the aim of maintaining the naturally protective concrete environment to manage corrosion.

The monitoring and testing protocol of constant current impressed current systems is well known [6]. However, given it is designed to measure polarisation rather than corrosion it is ill-suited to be a comprehensive protocol for constant voltage systems. This work will cover a scientific approach to monitoring the risk of corrosion both for constant current and constant voltage systems, both impressed current and galvanic, whether they be low maintenance simple patch repairs or intensively monitored systems for larger projects. Datadriven measurements are essential to ensuring that the corrosion in the area is prevented or managed in a satisfactory manner.

\section{Low maintenance systems:}

Patch repairs and low-risk projects may not need the intensive monitoring of a bridge pillar or car park. For these system designs, a simpler testing and monitoring protocol is used. This section constitutes the complete practice for low maintenance anode systems. One or many of the following surveying methods may be necessary based on the needs of the system and the client.

\subsection{Visual and delamination Surveys:}

All projects benefit from visual and delamination surveys in and around areas of corrosion risk and patch repairs at intervals agreed upon with the client that is consistent with the usage of the concrete structure. During the visual inspection, areas of moisture, staining, vandalism, cracking, exposed rebar, and discolouration should be noted. Any source of rust staining should be investigated. Cracks forming parallel to the rebar and spalling will be of particular concern. Visual surveys can be taken from a short distance or using binoculars if access is difficult.

Touching distance visual surveys are necessary if delamination is suspected or every six years (as noted in BD 63/17 [6]) for highway structures. These tests may include a "hammer tap" test for delamination in areas of concern found in the visual survey. These timelines can be adjusted according to access and risk, based on discussions between manufacturers, the appropriate agencies, and official inspectors. 


\subsection{Corrosion potential measurements:}

Measuring steel potentials against the potentials of a standard reference electrode (half-cell) is a wellestablished technique. In general measurements more positive than approximately $-200 \mathrm{mV}$ against $\mathrm{Cu} / \mathrm{CuSO}_{4}$ (copper/copper sulphate) are considered to be in the area of small corrosion risk and measurements more negative than $-350 \mathrm{mV}$ against $\mathrm{Cu} / \mathrm{CuSO}_{4}(\mathrm{CSE})$ are considered to be in the area of high risk of ongoing corrosion [7] [8]. Steel potentials can give a good approximation of the corrosion risk of steel, but they are affected by various other factors. Below are the shifts we expect to see in the potentials due to different environments in the concrete:

Tab. 1. Typical potential ranges measured in concrete using a Copper Sulphate reference electrode (V) [8]

\begin{tabular}{|l|l|}
\hline dry concrete & $0 \rightarrow+0.2$ \\
\hline dry, carbonated concrete & $0 \rightarrow+0.2$ \\
\hline humid, carbonated concrete & $-0.4 \rightarrow+0.1$ \\
\hline humid, chloride free concrete & $-0.2 \rightarrow+0.1$ \\
\hline wet, chloride contaminated concrete & $-0.6 \rightarrow-0.4$ \\
\hline $\begin{array}{l}\text { water saturated concrete without } \\
\text { Oxygen }\end{array}$ & $-1.0 \rightarrow-0.9$ \\
\hline
\end{tabular}

Different reference electrode materials will give different absolute potential measurements. It is therefore important to make a note of which is used during data collection.

Tab. 2. Relative potential against the standard hydrogen electrode for different half-cell materials (V) [8]

\begin{tabular}{|l|c|}
\hline \multicolumn{2}{|l|}{$\begin{array}{l}\text { Relative potentials against the standard hydrogen electrode } \\
\text { for different half-cell materials }(\mathrm{V})\end{array}$} \\
\hline Copper/Copper Sulphate saturated (CSE) & +0.318 \\
\hline Calomel $\left(\mathrm{Hg} / \mathrm{Hg}_{2} \mathrm{Cl}_{2}\right) \mathrm{KCl}$ saturated $(\mathrm{SCE})$ & +0.241 \\
\hline Silver Chloride $(\mathrm{Ag} / \mathrm{AgCl}) \mathrm{KCl}$ saturated & +0.199 \\
\hline
\end{tabular}

Tab. 3. ASTM Standard potentials and their corrosion risk [9]

\begin{tabular}{|l|l|l|}
\hline $\begin{array}{l}\text { Potential vs. } \\
\text { SCE }(\mathrm{mV})\end{array}$ & $\begin{array}{l}\text { Potential vs. } \\
\text { CSE }(\mathrm{mV})\end{array}$ & Probable Corrosion \\
\hline$>-123$ & $>-200$ & Low $(10 \%)$ risk \\
\hline-123 to -273 & -200 to -350 & Intermediate risk \\
\hline$<-273$ & $<-350$ & High $(90 \%)$ risk \\
\hline$<-421$ & $<-498$ & Severe corrosion \\
\hline
\end{tabular}

Manganese dioxide is also often used as the in-situ reference electrode given its stability, high $\mathrm{pH}$ and low chloride content.

\subsection{Potential Maps:}

A good way to reduce the effects of many of these potential shifts is to move away from taking individual potential measurements. Detailed potential maps consisting of corrosion potential measurements at close intervals (typically $50 \mathrm{~mm}$ ) may be undertaken at defined monitoring areas (limited in size) that are representative of a concrete structure or have a high risk of corrosion (ASTM 2015 [9]).
Corrosion potential or "half-cell" measurements can be taken with a wheel-based electrode or with a hand held electrode at set points between the $\mathrm{CP}$ anodes. The potentials are typically measured with reference to the potential of the reinforcement or, when not practical, with respect to another set position half-cell electrode [10]. This would reduce the need to damage concrete to expose the steel. These tests will be undertaken along the steel furthest from the sacrificial anodes, this steel is a relatively high risk given the lower protective current or using a line or two-dimensional grid (drawn onto the concrete) and will show localised changes in potential where anodes are present, as shown in Figure 1. This allows any possible anodic locations to be observed in the reinforcing steel as well as corroding galvanic anodes. Anodes indicate areas of active corrosion. Steel anodes represent actively corroding steel. Further investigation is then needed to see if this corrosion is in a controlled state or whether this is cause for concern.

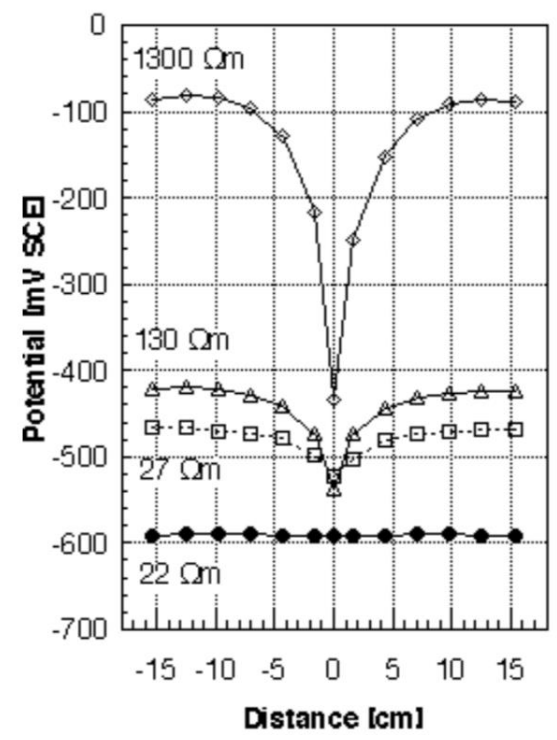

Fig. 1. half-cell potential model of anodic steel at different electrolyte resistivities in very wet, chloride containing mortar. Cover depth $20 \mathrm{~mm}$, anode $0.5 \mathrm{~cm}$. in very wet, chloride containing mortar. Rilem [8]

A detailed map of anode sites and historic potential map data are useful tools to see changes over time and to spot new or growing anodic sites for further investigation. Special attention should be given to the areas furthest from the anodes, as they will have the lowest protective current.

If no prior data is available, a visual assessment and an initial half-cell risk assessment over the structure or over representative areas should be used to find small areas of specific risk. These areas will be half-cell mapped alongside more typical areas and tracked throughout the remaining life of the structure.

To ensure good data is collected:

- Target steel locations within and immediately adjacent to any patch repairs. 
- ICCP systems should be turned off if possible, testing after 24-hours of depolarisation is preferred.

- There must be no coating or surface other than bare concrete.

- The voltmeter shall have at least $10 \mathrm{M} \Omega$ input impedance to limit the effect of meter impedance on the result.

- The surface must be wet to reduce the effect of moisture variation (see Table 1) and the half-cell electrode must make contact via a wet sponge.

- The sponge should be washed or replaced to avoid contaminants from the electrode affecting the measurement (sulphate and to a lesser extent chloride react with hydrated cement and change the potential).

- Use non-destructive techniques, like cover-meter mapping, to find the rebar if measurements are to be taken targeting specific steel elements. When steel locations are known a fast survey can be taken along the steel furthest from the sacrificial anodes.

- The calibration of all portable electrodes should be checked in a lab, and on-site against each other before use.

- Readings in an area specifically selected for monitoring must be recorded over the smallest practical distance (typically $50 \mathrm{~mm}$ ) to spot corrosion and increase the sensitivity of results. The size of the grid should be chosen so that there will be adequate points between known sacrificial anodes to identify an anode on the steel. A typical grid would have no more than $50-100 \mathrm{~mm}$ between measurement sites. These areas should be tested and tracked through accurate records for comparison during each scheduled evaluation.

- These regular test zones may be relatively small areas (typically $0.2-1 \mathrm{~m}^{2}$ ) that are at risk of corrosion and/or reprehensive of the structure.

The large amount of data collected per square meter when looking for anodes makes this technique only suitable for assessing limited, high-risk areas within a structure or failing that a few select representative sample locations of the structure. It is very useful however to ensure that what is being seen is corrosion, especially local pit corrosion and not a difference in moisture. Relative potentials show anode locations as sharp local changes in potential. A wheel anode increases the ease of this data collection. When doing a fast survey, the steel areas further from the anodes will be the most vulnerable to corrosion. Figure 2 (below) shows (1) typical steel rebar reinforcement with (2) regularly spaced anodes. Location (3) shows a desired minimal testing of the steel between the anodes which get the least protection and therefore at the highest risk. If a local anode is suspected, location (4) shows how two further measurements can ensure that the drop is a localised anode on the steel.

The criterion for success is simply that there are no anodic sites measured between or immediately around existing anodes. If anodic sites are found, they should be monitored over time to look for growth and any change in potential.

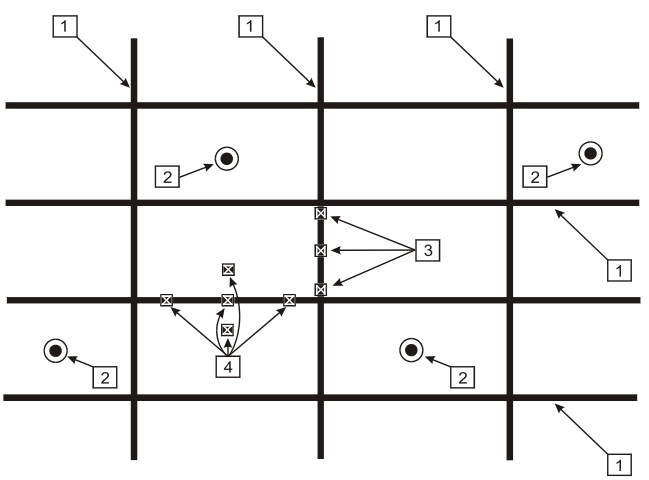

Fig. 2. Typical schematic showing rebar (1), anodes (2), primary half-cell testing (3) and secondary testing (4)

An example of a potential map is shown below:

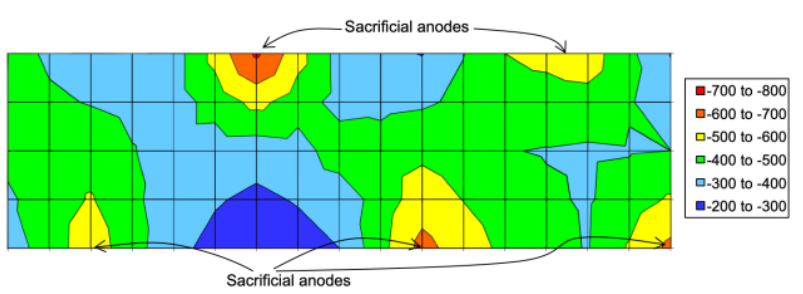

Fig. 3. Potential map of a structure containing embedded sacrificial anodes measured on a $50 \mathrm{~mm}$ grid. [8]

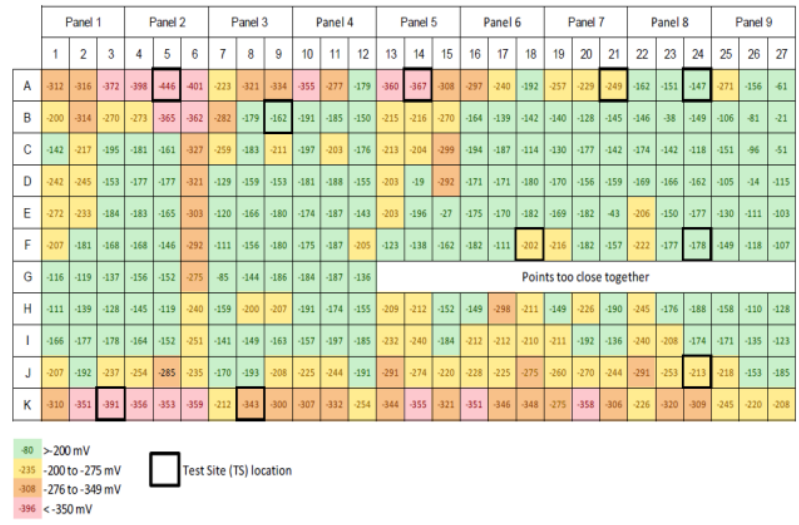

Fig. 4. Data taken from a bridge underpass before treatment. It can be used as a comparison for future half-cell tests. Areas that are red are at a higher risk of being an anode and would need further investigation.

In cases where a coating is to be applied, small coin sized areas can be ground down to give access or at least three embedded half-cell electrodes can be placed in the relatively high corrosion risk areas between anodes. Measurements can then be taken in the future between these electrodes, preferably using an electrode embedded at a lower risk site as a reference electrode.

Moisture differentials between reference and measurement electrodes can cause weak artificial anodes to occur on the map. This can be minimised by using reference electrodes in the same environment as the measurement and noting where mortar boundaries and other moisture boundaries are on the maps. Wetting the concrete surface and allowing it to permeate before measuring should also be standard practice to minimise this issue. 


\subsection{Current measurements}

This may be used on constant voltage ICCP and galvanic systems. Protective currents delivered by one or more galvanic anodes may be measured by installing a resistor (typically 1-10 Ohms) in the circuit between the anode(s) and the steel and then measuring the voltage across that resistor. A sensitive voltmeter, with a resolution of at least $0.1 \mathrm{mV}$, should be used to measure directly across the resistor to give the best data. Anodes with a higher current density are said to be more "active".

Galvanic anodes and constant voltage ICCP anodes often initially deliver a high current. The initial galvanic current off an anode is typically $3 \mathrm{~mA}$. The current decreases yearon-year after repairs due to many factors including; the concrete drying, the restoration of the concrete surrounding the steel, the creation of a benign and more resistive concrete environment, the build-up of resistive products around the anode, the reduction in galvanic anode size, and the galvanic anodes finding a local electrochemical equilibrium. The question of how we know the anodes are still active is one that troubles many in the field observing older systems.

During times of increased moisture or higher temperature the current output of the anodes increases. This is also when the steel is most vulnerable to corrosion. Significant transient increases in current have been measured. This is termed responsive behaviour because the anode responds to the corrosive nature of the environment [5]. We can use this behaviour to see if the system is still active.

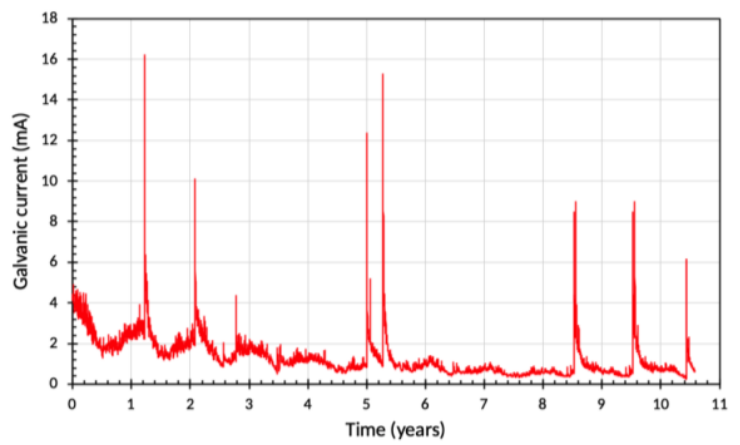

Fig. 5. Galvanic current from an anodic array over more than 10 years, showing the galvanic current response to flooding (tall peaks) and rainfall (smaller peaks) events.

Dry periods of low protective current are usually not a sign of the anodes depleting or disappearing. Galvanic systems work on a constant potential model so when the concrete is dry and stable, the steel is protected by the concrete. The anodes corrode less and maintain a lower protective current.

The higher initial current powers the migration of chloride ions away from the steel surface, the repassivation of the steel, and a lowering of the $\mathrm{pH}$ in the concrete around the reinforcement by the creation of hydroxide ions at the steel interface. To test whether the CP system is still "active, test the current flow after rain or during the day vs at night to see the responsive behaviour to increased ionic diffusion.
There is no minimum current criterion. In a completely benign concrete environment, the steel is protected without the application of any current.

ICCP systems have been turned off and yet years later corrosion has not yet become an issue because the environment has been restored and the steel is passive [11].

Figure 6 shows the anode current in response to a flooding event several years after installation. We can see that the anodes respond sharply to the changed environment and increased corrosion risk. The current then reduces as the concrete dries out over many weeks. Currents are higher in the zone with the highest moisture content.

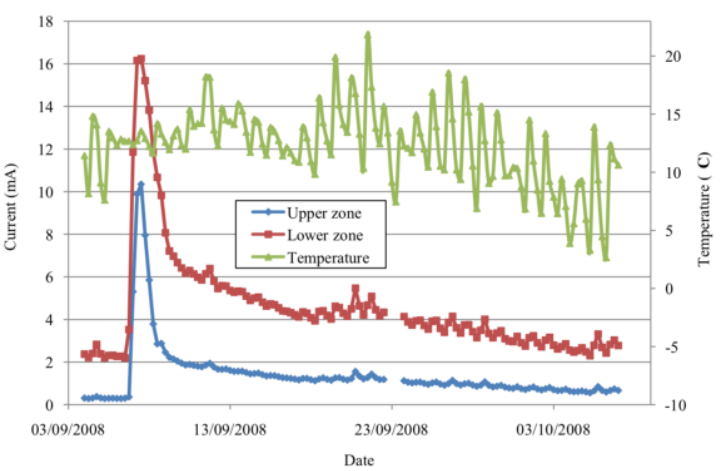

Fig. 6. Galvanic anode current response to flooding of Whiteadder Bridge, Northumberland, UK. [5]

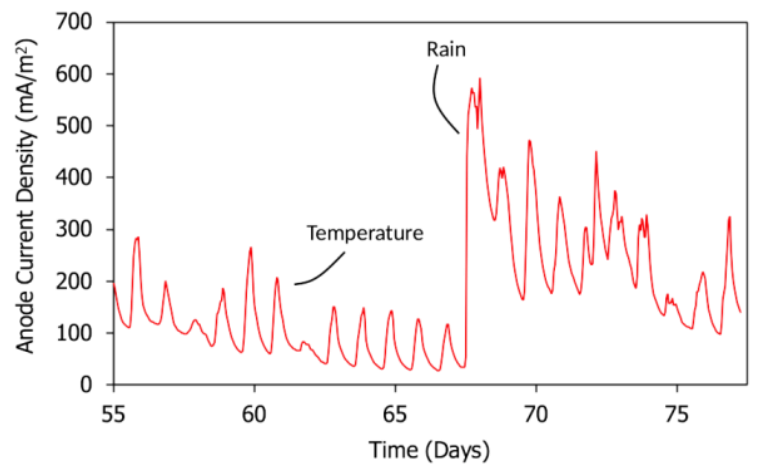

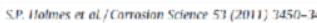

Fig. 7. Anode current density response to daily temperature oscillations and rainfall.

Figures 7 and 8 show how the current responds to temperature. Again, the current responds to the temperature dependent corrosion risk. The delay in response is attributed to the temperature recordings being atmospheric and the concrete taking a finite time to heat up. 


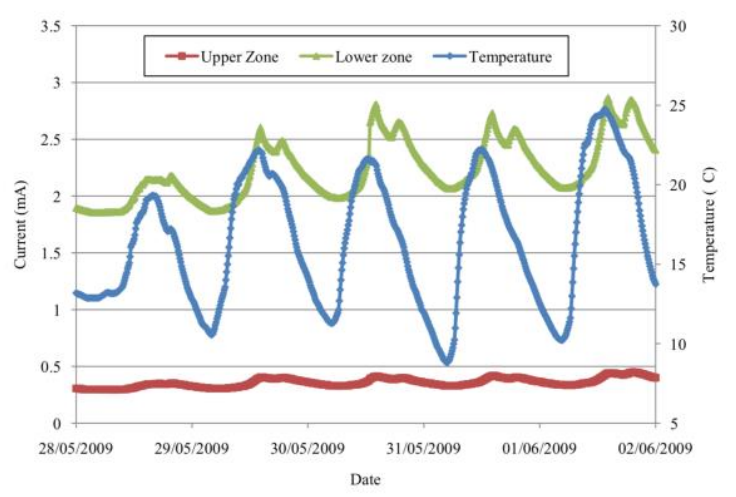

Fig. 8. Anode current response to temperature, Current and temperature data from a 5-day period in May/June 2009. [5]

The above constitutes the complete practice for low maintenance anode systems. What follows is an additional set of methods for intensively monitored, longlifetime systems.

\section{Intensively monitored systems with long design lives:}

The ISO BS EN 12696:2016 Standard has the following non-exhaustive criteria for achieving protection. No more than one must be met to meet the standard

a) an "Instantaneous OFF" potential more negative than $-720 \mathrm{mV}$ with respect to $\mathrm{Ag} / \mathrm{AgCl} / 0.5 \mathrm{M} \mathrm{KCl}$

b) from "Instantaneous OFF" a potential decay of at least $100 \mathrm{mV}$ over, at most, $24 \mathrm{hrs}$

c) potential decay over an extended period (typically over $24 \mathrm{hrs}$ ) of at least $150 \mathrm{mV}$ after "Instantaneous OFF". Use a reference electrode for any measurement over $24 \mathrm{hrs}$.

This data is taken via a polarisation test. Although this test is not supported by theoretical considerations it is seen as a non-exhaustive, practical standard. These criteria point towards the polarisation of the steel being adequate to achieve protection. In the case of galvanic and constantvoltage impressed current systems, protection is as a result of the re-establishment and maintenance of a protective concrete environment and this test methodology would be inappropriate.

An alternative set of performance verification criteria can be found in the notes of the ISO BS EN 12696:2016 standard and is permitted if none of the non-exhaustive list can be met. This entails a further assessment of corrosion. The following indicate a low risk

a) steel/concrete potential less negative than $-150 \mathrm{mV}$ with respect to $\mathrm{Ag} / \mathrm{AgCl} / 0.5 \mathrm{M} \mathrm{KCl}$ in a fully depolarised structure after the cathodic protection has been turned off (typically 7 days or more)

b) corrosion rates of less than $2 \mathrm{~mA} / \mathrm{m}^{2}$ or preferably less than $1 \mathrm{~mA} / \mathrm{m}^{2}$ to indicate passive steel.

c) a combination of a rising corrosion potential and falling corrosion rate.

The rest of this work will cover how to test to this combined standard. The focus will be on galvanic systems as these are the least understood, though all tests can be equally applied to constant-voltage impressed current systems that would also aim to restore the environment and benefit from this alternative standard.

\subsection{Polarisation decay and corrosion rate monitoring systems:}

Corrosion rate data should be collected from areas of the structure that are most at risk from further corrosioninduced deterioration. A visual inspection may identify areas that are most susceptible to further chloride contamination. Potential mapping may be used to identify areas of active corrosion. Areas historically known to be at high risk occur in the parent concrete adjacent to areas of concrete patch repair. A range of locations for monitoring should be selected to cover all risks.

\subsubsection{Polarisation decay measurements:}

When a current is applied to a metal, the potential changes. This potential shift is known as polarisation. By interrupting the current, the potential decays. The potential decay allows the calculation of a conservative estimate of the polarisation which in turn provides an indication of the passivation of the steel rebar.

A typical arrangement used to measure the potential shift in reinforced concrete that is treated with a hybrid electrochemical treatment is shown in Figure 9. With zinc anodes buried in cavities in the concrete. A reference electrode is located within the segment to determine the steel potential shift induced by the galvanic current. The rest of the anode system (the blue circuit in Figure 9) is used to minimize edge effects and confine the current from the anode segment to the steel below the segment (often termed a guard ring effect).

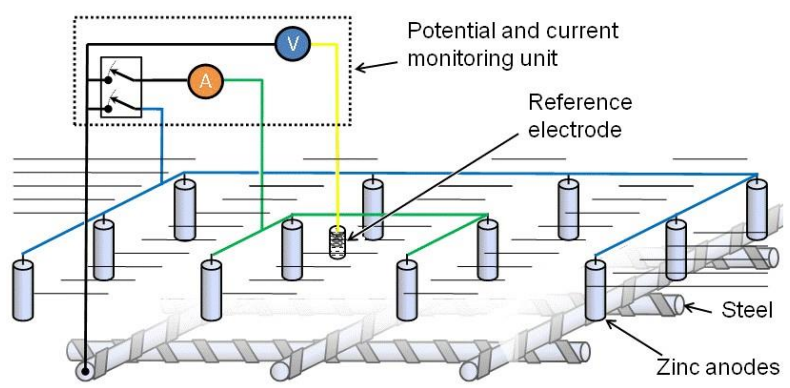

Fig. 9. Arrangement used to obtain data for corrosion rate calculation.

Polarisation potentials should be measured in the following way

- Remove galvanic link to turn off the system and instantly measure the steel/concrete potential, if the potential is more negative than $-720 \mathrm{mV}$ than it has passed, and no further test is needed

- Turn off for up to 24 hours and measure the steel/concrete potential. A shift in potential from the first measurement of $100 \mathrm{mV}$ is the minimum ISO standard, if passed no further measurement is needed 
- Turn off and measure the potential drop from the instant off potential over a longer time period, $150 \mathrm{mV}$ is the ISO standard.

This method is not based on theoretical models but is a known standard that has been used for some time. It measures the energy stored in the steel, or polarisation, which makes corrosion less energetically favourable and is dependent on the current supplied to the rebar by the anodes. For responsive systems such as hybrid galvanic anodes, once the concrete has dried and the steel environment re-alkalised the current needed to maintain the steel will often be too low to meet this standard, although the steel is being adequately protected.

\subsubsection{Corrosion rate measurements:}

If positive results are not possible with the previous test an alternative, a more scientifically rigorous measure, the corrosion rate, can be calculated. The basic method for this follows.

In Figure 9, the anode array is segmented so that the current density from a small segment of anodes (the green circuit in Figure 9) can be measured. This is used to determine the current density delivered to the steel by these anodes. The reference electrode should be located to see the average effects of the current (not the extremes due to variations in current distribution).

Rather than using an ammeter, insert a resistor in the galvanic circuit for each anode surrounding the reference electrode. After a few minutes, to let the current settle, use a voltmeter set in parallel with the resistor to measure the current off the segment of anodes. Using knowledge of the local steel density to calculate the applied current density per $\mathrm{m}^{2}$. From this the corrosion rate can be calculated. This relationship is given by the following equation.

$$
i_{\text {appl }}=i_{\text {corr }}\left(\exp \left(2.3 \Delta E / \beta_{c}\right)-\exp \left(-2.3 \Delta E / \beta_{a}\right)\right)
$$

$i_{\text {appl }}$ is the applied current density, $i_{\text {corr }}$ is the corrosion rate, $\Delta \mathrm{E}$ is the steel polarisation potential shift and $\beta_{a}$ and $\beta_{\mathrm{c}}$ are constants. This equation builds the basis for measuring the corrosion rate from the polarisation potential shifts. Values of $\beta_{a}$ and $\beta_{c}$ of $120 \mathrm{mV}$ were used in producing the current guidelines on the interpretation of corrosion rate data.

The following table can be used as a reference to analyse the results of this analysis.

Tab. 4. Corrosion rates and their relative risks taken from BRE Group [12]

\begin{tabular}{ll}
\hline Corrosion rate $\left[\mathrm{mA} / \mathrm{m}^{2}\right]$ & Risk \\
\hline$\leq 2$ & Very Low - Passive Steel \\
\hline $2-5$ & Low/Moderate \\
\hline $5-10$ & Moderate/High \\
\hline$\geq 10$ & High \\
\hline
\end{tabular}

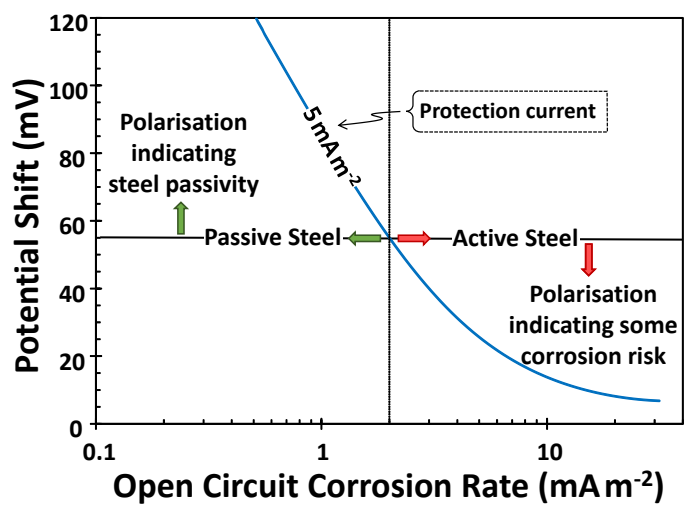

Fig. 10. Corrosion rate calculated as a function of potential shift at a current density of $5 \mathrm{~mA} / \mathrm{m}^{2}$ together with an example of its interpretation [13]

A large polarisation potential shift is induced by a small current density when the steel is passive. In the example in Figure 10, a potential shift of $55 \mathrm{mV}$ or more induced by a current density of $5 \mathrm{~mA} / \mathrm{m}^{2}$, would indicate that the steel is passive [13]. A falling corrosion rate and a rising corrosion potential also indicate that the system is providing protection and meets the alternative criterion allowed in ISO BS EN 12696:2016.

\subsection{Carbonation and chloride profiling:}

If an area appears to be corroding and the historic data shows the issue is getting worse, the last investigation before doing a destructive analysis of the area is testing the chemical make-up of the concrete around the rebar. Chloride sample standards are covered in ISO EN 14629:2012 and BRE Digest 444 Part 2:2000.

In areas of particular concern, following the assessment above, a semi-destructive chloride ion profile, can be used to test for the ingress of $\mathrm{pH}$ lowering ions near the target steel rebar. This will be done by drilling a $25 \mathrm{~mm}$ hole into the concrete and collecting the dust from different depths. The first $5 \mathrm{~mm}$ of concrete cover is discarded. Samples are then collected in 20 or $25 \mathrm{~mm}$ depth increments up to the rebar depth. These samples are then sealed and sent off to a lab for chemical analysis. Chloride amounts at the depth of and by the rebar of $0.4 \%$ or lower by weight of cement show a negligible to low probability of corrosion occurring as long as the concrete has not been carbonated. Ideally, the weight percentage will be $0.3 \%$ by weight of concrete or less (Bridge Advice Note 35 [14]).

Carbonation may be tested by applying phenolphthalein to a freshly taken core of concrete. If the result quickly shows a pink hue, then the concrete is still strongly alkaline, and carbonation has not reduced the $\mathrm{pH}$ to a dangerous level. If several minutes pass before the change in colour it may be a sign of some carbonation.

If a concrete structure has failed to meet this standard, then those high-risk areas will be in need of possible repair and/or further protection.

Rebar depth can and will vary over a structure. Technical drawings or a measurement in another location should not be taken as an accurate indication of the true 
rebar depth. Places with less concrete cover will be more likely to undergo higher levels of corrosion.

\section{Conclusion:}

Laid out above are the different tests that can be used to monitor old and new CP systems and how they meet with current ISO standards. Low galvanic current during dry periods should not be mistaken as a sign of the system underperforming if the corrosion risk remains low and the system responds to hazards with increased current output.

Using the alternative performance standards established in ISO EN BS 12696:2016 galvanic and constant-voltage impressed current systems can be held to a clear standard for the testing of corrosion risk.

Actions to take if a corrosion risk is identified include installing more galvanic anodes or applying a temporary electrochemical treatment (using the galvanic anodes, if possible) and attaching a temporary power supply (for hybrid systems). The options available will depend on the galvanic system used.

\section{References}

1. ISO: EN BS 12696 (2016).

2. R. F. Stratful, Progress report on inhibiting the corrosion of steel in a reinforces concrete bridge, Corr. (Jun. 1959).S. Beamish, Corrosion protection for reinforced concrete highway structures, [online] http://www.corrosionprevention.org.uk/Web\%20Do cs/Presentations $\% 20$ for $\% 20$ website/Manchester/Hig hway\%20Structures\%20-\%20Sam\%20Beamish.pdf (Sept. 2018)

3. H. Esteves, R. Brueckner, C. Atkins, T. Gerrard, and U. Hammer. Case studies of cathodic protection installations. [book auth.] J. Broomfield P. Chess. Cathodic Protection of Steel in Concrete and Masonry, 2nd Ed. CRC Press, (2013).

4. S. Holmes, G. Wilcox, P. Robins, G. Glass, and A. Roberts. Responsive behaviour of galvanic anodes in concrete and the basis for its utilisation. 10, Corr. Sci., 53, 3450-3454. ISSN 0010-938X (2010).

5. BD63/17, Highway Structures: Inspection and Maintenance of Highway Structures. Highways England, (2017).

6. P. Bamforth. Enhancing reinforced concrete durability Guidance on selecting measures for minimising the risk of corrosion of reinforcement in concrete. Concrete Society, (2004).

7. B. Elsener, C. Andrade, J. Gulikers, et al. Half-cell potential measurements-Potential mapping on reinforced concrete structures. 7, RILEM, Materials and Structures, 36, 461-471 (Aug. 2003).

8. ASTM C876-15, Standard Test Method for Corrosion Potentials of Uncoated Reinforcing Steel in Concrete. (2015).

9. C.S. TR60, Electrochemical tests for reinforcement corrosion. Concrete Society, (2004)
10. C. Christodoulou, G. Glass, J. Webb, S. Austin, C. Goodier, Assessing the long term benefits of Impressed Current Cathodic Protection, Corrosion Science, 52, Issue 8, 2671-2679. (2010).

11. BRE Digest 434, K. Hollinshead, Corrosion of reinforcement in concrete: Electrochemical monitoring. (Nov. 1998).

12. G.K. Glass, A.C. Roberts, N. Davison, Assessment of cathodic protection applied to above ground reinforced concrete, Materials and Corrosion, 70, 3, 503-510 (2019).

13. DMRB:3-3, BA 35/90, Inspection and repair of concrete highway structures (1990) 